\title{
A mixed formulation of mortar-based frictionless contact
}

\author{
I. Temizer* \\ Department of Mechanical Engineering, Bilkent University, 06800 Ankara, Turkey
}

\section{A R T I C L E I N F O}

Article history:

Received 18 July 2011

Received in revised form 13 February 2012

Accepted 23 February 2012

Available online 12 March 2012

\section{Keywords:}

Contact

Mortar method

Mixed formulation

Large deformation

\begin{abstract}
A B S T R A C T
A class of mortar-based frictionless contact formulations is derived based on a classical three-field mixed variational framework. Within a penalty regularization complemented by Uzawa augmentations, discrete mortar constraints are naturally induced by the variational setting. Major aspects of earlier mortar approaches are obtained through constrained, lumped or unconstrained recovery procedures for the mixed kinematic and kinetic mortar quantities from their projected counterparts. Two- and threedimensional examples at the infinitesimal and finite deformation regimes highlight the local and global quality of the contact interactions.
\end{abstract}

(c) 2012 Elsevier B.V. All rights reserved.

\section{Introduction}

Enforcing contact constraints, even in the absence of friction, poses challenges that have been the source of significant research in finite element analysis. Currently, mortar-based contact treatment forms the widely accepted state-of-the-art class of approaches. Emanating from the mathematical literature, the presentation of these approaches have first carried over into the engineering community in a precise form with detailed statements of various discretization restrictions. In subsequent works, some of these restrictions have been dropped and the presentation has evolved towards a more practical form. On the other hand, several of the fundamental ingredients of mortar approaches have first appeared in the engineering literature in the context of mixed formulations for frictionless contact. While a mixed formulation concept is implicit in a mortar-based treatment, an explicit link between these two classes of approaches has not been demonstrated in the literature. The primary goal of the present work is to reconcile these approaches by investigating the link for frictionless contact. In doing so, various mortar formulations from the mathematical and engineering works will be recovered with variations whose advantages and disadvantages will be discussed. Care is taken to mention relevant key contributions, presently concentrating on frictionless contact modeling. However, a review of the broad field of contact mechanics is not attempted. In particular, a large class of contact algorithms that emanate from the node-tosegment (NTS) approach and which have important applications in a variety of practical cases is not referenced. The reader is referred

\footnotetext{
* Tel.: +90 3122903064 .

E-mail address: temizer@bilkent.edu.tr
}

to $[23,47]$ for extensive references on such approaches. Additionally, a treatment of one-dimensional manifolds is also not considered - cf. [22].

Mixed formulation ideas have originated in the context of volumetric problems for solids and fluids, for instance in order to avoid an over-constrained pressure approximation based on the classical Veubeke-Hu-Washizu variational principle $[42,36]$. The introduction of mixed formulations in contact problems goes back to the works of [37] where a perturbed Lagrangian approach was constructed to enforce the contact constraints and [29] where a pure penalty enforcement was employed. In both of these works, the pressure was assigned an independent discontinuous discretization that was inherited from a segmentation of the contact interface based on the geometry of the non-matching meshes. Subsequently, the contact constraints were enforced in a weak sense within each contact segment. It is remarked that the augmented Lagrangian treatment introduced in [1], as further investigated in [30] and closely related to [16], was originally designed as a mixed treatment of penalty and Lagrange multiplier approaches. In this work, the terminology mixed refers to the three-field approach of [29], which will be taken as the starting point. Moreover, emphasis will be placed on the discretization of the constraints rather than the particular numerical method with which they are enforced. In particular, a penalty enforcement will be pursued together with Uzawa augmentations to achieve robustness. In this context, the presence of other mixed methods suitable for contact analysis should also be mentioned $[25,15]$.

Mortar methods were first introduced in the context domain decomposition to address the coupling of interfaces with nonmatching meshes. See [46] for an overview and [32,11,43,44] for recent developments. Early applications in contact mechanics go 
back to [2,13]. The importance of a mortar-based treatment of interface constraints are twofold. First, the method ensures that the flat interface patch test of [29] is satisfied, which indicates a correct transmission of the interface pressure. The correct transmission is realized by an appropriate discretization of the pressure field complemented by an accurate evaluation of the contact integrals that is typically realized through the introduction of an intermediate surface which is constructed via segmentation. Second, mortar based approaches do not display surface locking when the interfaces are curved $[28,20]$ so that, in the context of the penalty enforcement of the constraints, Uzawa augmentations of the pressure converge [38]. This is ensured by enforcing a suitable number of constraints such that the interfaces are able to conform to each other in order to satisfy them. The classical one-pass NTS algorithm does not pass the patch test although it naturally delivers the correct number of constraints, while the two-pass NTS approach has the reverse qualities. Additionally, a well-defined interface pressure does not exist for the NTS algorithm, requiring classical tributary area type post-processing techniques to obtain a local pressure. A straightforward technique of working with a local pressure is by enforcing the constraints pointwise in the evaluation of the contact integrals which, however, has performance qualities similar to the two-pass NTS scheme.

Early applications of the mortar method to small deformation contact problems of engineering interest parallel the developments in the mathematical literature [27]. Subsequent developments in $[33,18,17,7,31,10,19,3,12]$ have extended this approach to the finite deformation regime with large sliding where non-matching meshes naturally occur and have investigated various aspects of discretization and integration in space and time. It was found out that, in addition to the ability to pass the patch test while avoiding surface locking, mortar-based approaches are able to tackle problems that were previously found challenging using the classical NTS algorithm and their variants. There are three main ingredients in a mortarbased treatment of contact in a frictionless setting: (i) discretization of the pressure field on the non-master surface, (ii) formulation of a discrete number of constraints in terms of projected kinematics and (iii) an accurate evaluation of the contact constraints. While the segmentation approach delivers high accuracy $[33,12]$, a direct integration on the non-mortar surface without explicit segmentation was also observed to deliver satisfactory performance [7,41]. The projected kinematics constructs an operator that maps the positions of the mortar surface onto the non-mortar side discretization. This can be obtained either by first constructing a discrete gap vector and subsequently obtaining a scalar gap $[13,33]$ or by directly operating on the scalar gap distribution $[41,40]$. For both items, the latter choices will be taken as the starting point. Finally, the discretization of the pressure field governs the stability and convergence properties of the contact algorithm. The simplest (standard) choice in the literature that delivers a satisfactory performance, which also appears in alternative formulations [6], is inheriting the basis functions of the non-mortar surface. Special restrictions are typically imposed on the pressure field at the edge of the contact interface $[13,18]$ although these can be safely dropped in a broad range of examples $[33,41,10]$. When Lagrange multipliers or the augmented Lagrangian approach is employed, dual basis functions [45] may be constructed to locally condense out the multipliers to alleviate the computational cost $[46,19]$ but other choices are also possible [8]. The pressure discretization and the projected kinematics together deliver a set of discrete constraints which preserve the integration of a pressure distribution but enforce the continuum constraints in a weak sense, in line with the ideas in $[37,29]$. It is remarked that a straightforward application of mortar approaches may also deliver poor results, as demonstrated for a slave surface that is significantly coarser than the master [8] and recently in the context of enriched interfaces [24]. Presently, standard basis functions will be employed to discretize the pressure distribution. It is remarked that, although not classified as mortar, the series of works by $[28,20,39,38]$ incorporate all the essential features of mortar-based treatments, with additional emphasis on minimizing, or eliminating when possible, the geometric bias [29] that is associated with the designation of master/slave or mortar/non-mortar. The geometric bias, although less pronounced compared to the NTS approach, still exists in the mortar-based treatments $[33,34]$ but two-pass mortar approaches have also been developed to alleviate this effect [35].

In order to recover various aspects of mortar approaches from a mixed treatment, the classical three-field mixed formulation of [29] is investigated in Section 2. Here, the continuum formulation is followed by a mortar-based relaxation of the contact constraints through appropriate projections of the mixed kinematic and kinetic variable distributions. The induced weak formulation delivers three possibilities for the recovery of the discretized mixed variables: (i) unconstrained, (ii) lumped and (iii) constrained. The consistency requirements for the implementation of these approaches within an Uzawa augmentation scheme are discussed and finally the linearization of the overall algorithm is addressed in Section 3 where it is additionally observed that the symmetry properties of the original continuum formulation are preserved with the constrained and lumped recovery approaches. Representative numerical investigations in Section 4 demonstrate the performance of each recovery approach for both two- and three-dimensional examples in the infinitesimal and finite deformation regimes. The quality of the solutions are assessed through local pressure distributions that are compared with analytical solutions where possible as well as by monitoring the smoothness of global structural forces.

\section{Three-field mixed normal contact treatment}

\subsection{Continuum formulation and finite element discretization}

Let $\mathcal{R}_{0}$ and $\mathcal{R}$ denote the reference and current configurations of a material body $\mathcal{B}$, with respective position vectors $\boldsymbol{X}$ and $\boldsymbol{x}$. The configurations are related by the motion $\boldsymbol{x}=\boldsymbol{\chi}(\boldsymbol{X})$ that induces the deformation gradient $\boldsymbol{F}=\operatorname{Grad} \boldsymbol{\chi}$. In a finite deformation framework, the strong form of the linear momentum balance is

$\operatorname{Div}[\boldsymbol{P}]=0 \quad$ in $\mathcal{R}_{0}$

where $\boldsymbol{P}$ is the first Piola-Kirchhoff stress tensor and, introducing $\boldsymbol{N}$ as the outward unit normal to the boundary $\partial \mathcal{R}_{o}, \boldsymbol{p}=\boldsymbol{P N}$ is the associated Piola traction. The corresponding boundary value problem is subject to the boundary conditions

$\boldsymbol{x}=\hat{\boldsymbol{x}} \quad$ on $\quad \partial \mathcal{R}_{o}^{x} \quad$ and $\boldsymbol{p}=\hat{\boldsymbol{p}}$ on $\partial \mathcal{R}_{o}^{p}$.

In order to model the unilateral contact between two bodies, let $\mathcal{B}^{(1)}$ be the slave (non-mortar/contactor) and $\mathcal{B}^{(2)}$ the master (mortar/target) side. The position vector $\boldsymbol{x}$ will be reserved for the slave side while the master side will be distinguished by $\boldsymbol{y}$. The matching contact interface $\partial \mathcal{R}^{c}:=\partial \mathcal{R}^{(1), c}=\partial \mathcal{R}^{(2), c}$ on the deformed configuration is pulled back to $\partial \mathcal{R}_{o}^{c}:=\partial \mathcal{R}_{o}^{(1), c} \neq \partial \mathcal{R}_{o}^{(2), c}$. All contact integrals are subsequently evaluated on $\partial \mathcal{R}_{0}^{c}$, which will also denote the designated potential contact portion of the slave surface in the discrete setting. The weak form of the linear momentum balance is then expressed as

$\delta \mathcal{G}:=-\sum_{I=1}^{2} \int_{\mathcal{R}_{o}^{(I)}} \delta \boldsymbol{F} \cdot \boldsymbol{P} \mathrm{d} V+\sum_{I=1}^{2} \int_{\partial \mathcal{R}_{o}^{(I), p}} \delta \boldsymbol{x} \cdot \hat{\boldsymbol{p}} \mathrm{d} A+\int_{\partial \mathcal{R}_{0}^{c}}(\delta \boldsymbol{x}-\delta \boldsymbol{y}) \cdot \boldsymbol{p} \mathrm{d} A=0$

where $\boldsymbol{p}:=\boldsymbol{p}^{(1)}$. In this work, frictionless contact is considered. Consequently, the contact traction is $\boldsymbol{p}=p_{N} v$ where $v$ is the outward unit normal to $\partial \mathcal{R}_{o}^{(2), c}$. Using the standard definition 
$g_{N}=-(\boldsymbol{x}-\boldsymbol{y}) \cdot v$ for the normal gap, the normal contact contribution to the weak form can be expressed as

$\delta \mathcal{G}^{c}:=\int_{\partial \mathcal{R}_{0}^{c}}(\delta \boldsymbol{x}-\delta \boldsymbol{y}) \cdot \boldsymbol{p} \mathrm{d} A=-\int_{\partial \mathcal{R}_{o}^{c}} \delta g_{N} p_{N} \mathrm{~d} A=: \delta \mathcal{G}_{N}^{c}$.

The local form of the Hertz-Signorini-Moreau, or KarushKuhn-Tucker optimality, conditions for impenetrability constraints on $\partial \mathcal{R}_{o}^{c}$ are

$g_{N} \leqslant 0, \quad p_{N} \geqslant 0, \quad g_{N} p_{N}=0$.

It is emphasized that $\boldsymbol{y}$ is defined through the closest-point projection of a slave point onto the master surface, although this dependence is not explicitly denoted for notational brevity. Therefore, quantities such as $\delta \boldsymbol{y}$ should be interpreted carefully as $\boldsymbol{y}$ is not entirely independent of $\boldsymbol{x}$.

\subsection{Mixed formulation}

Within a penalty regularization of the contact constraints, the normal contact contribution to the weak form emanates from the variation of

$\mathcal{G}_{N}^{c}:=-\frac{\epsilon_{N}}{2} \int_{\partial \mathcal{R}_{0}^{c}} g_{N}^{2} \mathrm{~d} A$

such that $p_{N}=\epsilon_{N} g_{N}$. On the other hand, introducing a mixed kinematic variable $\gamma_{N}$, the classical three-field mixed formulation in terms of $\left\{g_{N}, \gamma_{N}, p_{N}\right\}$ takes the starting point as the variation of the functional [29]

$\mathcal{C}_{N}\left[g_{N}, \gamma_{N}, p_{N}\right]:=-\frac{\epsilon_{N}}{2} \int_{\partial \mathcal{R}_{0}^{c}} \gamma_{N}^{2} \mathrm{~d} A+\int_{\partial \mathcal{R}_{0}^{c}} p_{N}\left(\gamma_{N}-g_{N}\right) \mathrm{d} A$

which delivers three terms:

$$
\begin{aligned}
\delta \mathcal{C}_{N}= & -\int_{\partial \mathcal{R}_{o}^{c}} \delta g_{N} p_{N} \mathrm{~d} A+\int_{\partial \mathcal{R}_{o}^{c}} \delta p_{N}\left(\gamma_{N}-g_{N}\right) \mathrm{d} A \\
& +\int_{\partial \mathcal{R}_{0}^{c}} \delta \gamma_{N}\left(p_{N}-\epsilon_{N} \gamma_{N}\right) \mathrm{d} A
\end{aligned}
$$

Here, the first term is the contribution $\delta \mathcal{G}_{N}^{c}$ to the linear momentum balance. The independent variations $\delta p_{N}$ and $\delta \gamma_{N}$ in the two remaining terms induce the equalities

$\int_{\partial \mathcal{R}_{0}^{c}} \delta p_{N} \gamma_{N} \mathrm{~d} A=\int_{\partial \mathcal{R}_{0}^{c}} \delta p_{N} g_{N} \mathrm{~d} A$

and

$\int_{\partial \mathcal{R}_{o}^{c}} \delta \gamma_{N} p_{N} \mathrm{~d} A=\epsilon_{N} \int_{\partial \mathcal{R}_{o}^{c}} \delta \gamma_{N} \gamma_{N} \mathrm{~d} A$

that would deliver the identifications $\gamma_{N}=g_{N}$ and $p_{N}=\epsilon_{N} \gamma_{N}=\epsilon_{N} g_{N}$ in the continuum setting where a pointwise satisfaction of the constraint $g_{N}=0$ on $\partial \mathcal{R}_{o}^{c}$ is possible.

\subsection{Mortar-based relaxation of the constraints}

The discretizations of the slave and master surfaces are respectively denoted by

$\boldsymbol{x}=\sum_{I} N^{I} \boldsymbol{x}^{I}$ and $\boldsymbol{y}=\sum_{J} M^{J} \boldsymbol{y}^{J}$,

with $\left\{\boldsymbol{x}^{I}, \boldsymbol{y}^{I}\right\}$ as the nodal positions and $\left\{N^{I}, M^{J}\right\}$ as the basis functions. In the discrete setting, a direct application of $p_{N}=\epsilon_{N} g_{N}$ leads to an over-constrained formulation if enforced directly at each point of the slave surface $\partial \mathcal{R}_{o}^{c}$. Consequently, one proceeds by admitting a discretization of the mixed kinematic variable $\gamma_{N}$ and the mixed kinetic variable $p_{N}$. Presently, where an analogy to existing penalty-regularized mortar formulations is desired, the presentation deviates from the original developments in [29] and this discretization is inherited from the discretization of the slave surface via [33]

$p_{N}=\sum_{I} N^{I} p_{N}^{I} \rightarrow \delta p_{N}=\sum_{I} N^{I} \delta p_{N}^{I}$

and

$\gamma_{N}=\sum_{I} N^{I} \gamma_{N}^{I} \rightarrow \delta \gamma_{N}=\sum_{I} N^{I} \delta \gamma_{N}^{I}$

whereas the purely kinematic quantity $g_{N}$ does not admit such a discretization in general. As mentioned earlier, additional restrictions at the edge of the contact interface have been dropped in this discretization $[13,33,18]$. Introducing the notations

$\langle\bullet\rangle:=\int_{\partial \mathcal{R}_{0}^{c}}(\bullet) \mathrm{d} A$ and $\overline{(\bullet)^{I}}:=\left\langle N^{I}(\bullet)\right\rangle$,

the right-hand side identity defining the mortar projection, Eq. (2.9) can be rewritten as

$\left\langle\sum_{I} \delta p_{N}^{I} N^{I} \gamma_{N}\right\rangle=\left\langle\sum_{I} \delta p_{N}^{I} N^{I} g_{N}\right\rangle$

which implies

$\bar{\gamma}_{N}^{I}=\bar{g}_{N}^{I}$

Clearly, $\gamma_{N}$ cannot be pointwise equal to $g_{N}$ in general since the former inherits the discretization of the slave surface only whereas the latter varies according to the discretizations of both the slave and the master surfaces. Similarly, Eq. (2.10) implies

$\bar{p}_{N}^{I}=\epsilon_{N} \bar{\gamma}_{N}^{I}=\epsilon_{N} \bar{g}_{N}^{I} \leftrightarrow p_{N}^{I}=\epsilon_{N} \gamma_{N}^{I}$.

In the upcoming discussion, the discrete variables $\bar{p}_{N}^{I}$ and $\bar{g}_{N}^{I}$ will be referred to as the projected mortar quantities whereas $p_{N}^{I}$ and $\gamma_{N}^{I}$ will be referred to as the mixed mortar quantities.

The central ingredient of a mortar-based constraint treatment is the weak enforcement

$\int_{\partial \mathcal{R}_{0}^{c}} p_{N} g_{N} \mathrm{~d} A=\sum_{I} p_{N}^{I} \bar{g}_{N}^{I}=\sum_{I} p_{N}^{I} \bar{\gamma}_{N}^{I}=\sum_{I} \bar{p}_{N}^{I} \gamma_{N}^{I} \stackrel{\lim _{N} \rightarrow \infty}{\longrightarrow} 0$.

However, as will be identified shortly, the determination of $\gamma_{N}^{I}$ and consequently that of $p_{N}^{I}$ requires a knowledge of the (discretized) contact area $\partial \mathcal{R}_{o}^{c}$ in general. Consequently, the mortar counterpart of the Hertz-Signorini-Moreau conditions are expressed in terms of the projected quantities:

$\bar{g}_{N}^{I} \leqslant 0, \quad \bar{p}_{N}^{I} \geqslant 0, \quad \bar{g}_{N}^{I} \bar{p}_{N}^{I}=0$.

The set of nodes for which $\bar{p}_{N}^{I} \geqslant 0$ defines the active set $\mathcal{A}$. While $\bar{g}_{N}^{I}>0$ updates the status of an inactive node to active, $\bar{p}_{N}^{I}<0$ is used to deactivate the contact status since an augmented Lagrange multiplier setting in the context of the Uzawa method will eventually be employed. The discrete contact area is composed of all elements which have at least one active node. It is remarked that requirement (2.19) is implied by (2.18) provided vanishing $\bar{g}_{N}^{I}$ imply vanishing $\gamma_{N}^{I}$. This is shown in the following section.

\subsection{Recovery of the mixed mortar quantities}

In order to recover the kinematic mixed mortar quantity $\gamma_{N}^{I}$, which defines the kinetic term $p_{N}^{I}$ via Eq. (2.17), $\gamma_{N}^{I}$ is set to zero for all inactive points. Introducing the indicator

$\chi^{I}= \begin{cases}1 & \text { if } I \in \mathcal{A} \\ 0 & \text { otherwise }\end{cases}$

and the mortar overlap integrals

$\Phi^{I J}:=\left\langle N^{I} N^{J}\right\rangle$,

the system of equations for $\gamma_{N}^{I}$ emanating from Eq. (2.16), which is now more appropriately stated as $\bar{\gamma}_{N}^{I}=\chi^{I} \bar{g}_{N}^{I}$, are 
$\sum_{J} \underbrace{\chi^{I} \Phi^{I J} \chi^{J}}_{=: \Psi^{I I}} \gamma_{N}^{J}=\sum_{J} \Psi^{I J} \gamma_{N}^{J}=\chi^{I} \bar{g}_{N}^{I} \quad \forall I$.

Here, $\Psi^{I J}$ has been introduced to highlight that the solution of these equations requires a consideration of only those nodes which are active and the sum over $J$ is understood to be taken only over these. $\Phi^{I I}$ is a symmetric positive-definite matrix. ${ }^{1}$ The rows and columns of $\Psi^{I J}$ associated with the active nodes also form a symmetric positive-definite submatrix. The corresponding inverse submatrix is appended by rows and columns of zeroes to incorporate inactive nodes and is denoted by $\widehat{\Psi}^{I I}$. Consequently, one may write

$\gamma_{N}^{I}=\sum_{J} \widehat{\Psi}^{I J} \chi^{J} \bar{g}_{N}^{J}$

It is highlighted again that the choice of vanishing $\gamma_{N}^{I}$ for $I \notin \mathcal{A}$ is enforced explicitly here. Since the inverse $\widehat{\psi}^{I I}$ exists, vanishing $\bar{g}_{N}^{I}$ imply vanishing $\gamma_{N}^{I}$. However, although $\bar{g}_{N}^{I}=\bar{\gamma}_{N}^{I} \geqslant 0$ is guaranteed, $\gamma_{N}^{I}<0$ is possible. Consequently, $p_{N}<0$ is possible on $\partial \mathcal{R}_{o}^{c}$ in a mortar setting while $g_{N}<0$ is naturally allowed in the evaluation of $\bar{g}_{N}^{I}$.

This completes the treatment of normal contact apart from linearization, which is treated in Section 3. The presented recovery procedure is chosen as the default one due to various advantages it possesses and designated as constrained. It is remarked that the resulting system of equations for $\gamma_{N}^{I}$ closely resembles the procedure of [13]. However, therein the mortar projection is obtained by first constructing a discrete gap vector and subsequently obtaining a scalar gap, as opposed to choice of directly operating on the scalar gap distribution. Next, alternative recovery procedures are discussed.

\subsection{Alternative recovery procedures}

\subsubsection{Unconstrained recovery}

The formulation of Section 2.4 enforces the condition $\gamma_{N}^{I}=0$ for $I \notin \mathcal{A}$. As will be demonstrated in Section 3, this choice ensures the symmetry of the tangent matrix emanating from the normal contact formulation. This advantage is accompanied by numerical efficiency due to a reduced set of slave elements over which the contact integrals need to be evaluated. However, as will be observed in Section 4, this convenience has the drawback that smooth changes in $\bar{g}_{N}^{I}$ are not always accompanied by smooth changes in $\gamma_{N}^{I}$ when there is significant reduction in the active set. Consequently, at coarse discretizations, local and global contact interactions may experience jumps. An unconstrained recovery procedure is based on

$\sum_{J} \Phi^{I J} \gamma_{N}^{J}=\chi^{I} \bar{g}_{N}^{I} \quad \forall I \rightarrow \gamma_{N}^{I}=\sum_{J} \widehat{\Phi}^{I J} \chi^{J} \bar{g}_{N}^{I}$

where $\widehat{\Phi}^{\text {IJ }}$ are associated with the inverse of $\Phi^{I J}$. Since the resulting $\gamma_{N}^{I}$ are generally non-zero even for $I \notin \mathcal{A}$, all slave elements contribute to the contact integrals at all times, which results in a higher computational effort in comparison with the default formulation. In other words, the discrete contact area is always equivalent to the whole designated potential contact portion of the slave surface. This formulation additionally leads to unsymmetric tangent matrices. However, smooth evolutions of the contact interactions are ensured even at coarse discretizations. Clearly, the differences between the constrained and unconstrained formulations vanish when all nodes are active. It is also remarked that the expense of matrix inversion in these two formulations is minimal compared to other stages of computation.

\footnotetext{
1 Matrices are referred to by their components without introducing additional notation.
}

\subsubsection{Lumped recovery}

Approximate recovery procedures can be shown to capture the main features of various earlier mortar implementations. One starting point is a simplification of $\widehat{\Psi}^{I J}$ in (2.23). A natural approach is a diagonal approximation to $\Psi^{I J}$ for which row-sum lumping is the simplest choice. This delivers the diagonal components

$\psi_{1}^{I}:=\sum_{J} \Psi^{I J}$

for the approximating matrix. A second approach would take (2.24) as the starting point. When combined with the partition of unity of the contact discretization, this choice delivers the result

$\psi_{2}^{I}:=\sum_{J} \Phi^{I J}=\left\langle N^{I}\right\rangle$.

The resulting mortar formulation indeed closely resembles the choices in $[33,41]$ but presently also ensures the symmetry of the linearization. The use of either $\psi^{I}$ definition leads to a simplified lumped recovery of the mixed kinematic variable:

$\gamma_{N}^{I}=\chi^{I} \frac{\bar{g}_{N}^{I}}{\psi^{I}}$

Without augmentations, which will be pursued in the next section, either $\psi^{I}$ delivers similar results. However, the computation of $\psi_{1}^{I}$ requires a knowledge of the active set, which will be found inconvenient within the augmentation setting. For this reason, $\psi^{I}$ will refer to the second method. It is remarked that either definition is guaranteed to deliver non-negative mixed quantities whenever $\psi^{I}>0$. In general, however, lumping may lead to negative $\psi^{I}$ when the basis functions are not everywhere non-negative. The use of such basis functions has been investigated in [14,35]. Linear Lagrange or quadratic NURBS basis functions that are employed in this work are everywhere non-negative and hence guarantee $\psi^{I}>0$.

It is remarked that a particular approach that simplifies the implementation within a Lagrange multiplier setting is to admit an independent discetization of the mortar variables by not employing $N^{I}$ but dual basis functions which are orthogonal to $N^{l}$ such that $\Psi^{I I}$ is diagonalized $[45,19]$, similar to lumped recovery.

\subsection{Augmentation within Uzawa iterations}

Choosing a large value of $\epsilon_{N}$ often does not lead to a robust performance of contact algorithms [50], in particular at large initial penetrations [49]. Moreover, it is not a straightforward task to assess the correct value of $\in_{N}$. In particular, as $\epsilon_{N}$ is increased towards the Lagrange multiplier limit beyond a region where the solution might already be qualitatively satisfactory, an unstable formulation will start displaying oscillations that grow in magnitude with $\in_{N}$. In order to both ensure a robust and accurate formulation while checking for stability numerically, the augmented Lagrange multiplier method will be employed in the context of Uzawa iterations in view of its implementation simplicity - cf. [30]. Uzawa iterations are easily observed not to converge at small tolerances for unstable formulations due to surface locking [38].

\subsubsection{Consistency statement}

Augmentation of the mortar variables are naturally carried out through projected quantities. Denoting with the superscript $k$ the Uzawa augmentation step at time/load step $n+1$, the projected pressures are updated via

$\bar{p}_{N}^{I}=\bar{p}_{N}^{I,(k)}+\epsilon_{N} \bar{g}_{N}^{I}$

which are subsequently employed in updating the active set, where unindexed quantities are understood to belong to augmentation step $k+1$. Now, returning to an explicit statement of Eq. (2.17) as a system of equations in the form 
$p_{N}^{I}=\sum_{J} \widehat{\Psi}^{I J} \chi^{J} \bar{p}_{N}^{J}$

a recovery of the mixed pressure is based on the consistency statement $\left(p_{N}^{I}=0\right.$ if $\left.I \notin \mathcal{A}\right)$

$p_{N}^{I}=p_{N}^{I,(k)}+\epsilon_{N} \gamma_{N}^{I}$.

In other words, the augmented pressures are subject to the already defined mortar projection:

$p_{N}^{(k)}=\sum_{J} N^{J} p_{N}^{J,(k)} \rightarrow \bar{p}_{N}^{I,(k)}=\left\langle N^{I} p_{N}^{(k)}\right\rangle$

In the implementation of this algorithm, either the mixed pressures or the projected ones are transmitted from one augmentation step to the other and the remaining quantity needs to be recovered by projection or through the solution of the system of equations, respectively. The augmentations are continued until an iterative error measure $£^{(k+1)}$, to be specified in Section 4 , satisfies a convergence criterion to within a given tolerance ToL:

$\mathfrak{£}^{(k+1)} \leqslant$ TOL.

\subsubsection{Alternative treatments}

In the case of lumped recovery, one obtains the expression

$p_{N}^{I,(k)}=\frac{\bar{p}_{N}^{I,(k)}}{\psi^{I}}$,

which is not consistent with Eq. (2.31). Consequently, it is necessary to define, when $p_{N}^{I,(k)}$ is the stored variable,

$\bar{p}_{N}^{I,(k)}=p_{N}^{I,(k)} \psi^{I}$.

This modification also ensures that $p_{N}^{I,(k)}$ remains positive whenever $\psi^{I}>0$. When not employed, the inconsistency leads to a loss of quadratic convergence within an augmentation step and may also cause the discrete pressures to change through augmentations at a fixed active set with vanishing discrete gap when they should trivially remain constant. Since the active set is updated via $\bar{p}_{N}^{I}, \psi_{1}^{I}$ cannot be employed in this setting - see Section 2.5.2. It is remarked that although it does not explicitly appear in the formulation $\bar{\gamma}_{N}^{I}$ is also subject to the same consistency statement. However, this is trivially satisfied by (2.27).

In the case of an unconstrained recovery, the distribution $p_{N}^{(k)}$ from the last augmentation should be updated to account for changes in the active set to ensure consistency among the mixed and projected quantities associated with the augmented pressure distribution $p_{N}$. For this purpose, in a first step the projected quantities $\bar{p}_{N}^{I,(k)}$ are computed via (2.31) and subsequently the mixed quantities $p_{N}^{I,(k)}$ are updated via

$p_{N}^{I,(k)}=\sum_{J} \widehat{\Phi}^{I J} \chi^{J} \bar{p}_{N}^{I,(k)}$

Clearly, the input and output values of $p_{N}^{I,(k)}$ are the same if there are no changes in the active set. Since $\gamma_{N}^{I}$ is generally non-zero for $I \notin \mathcal{A}$ with unconstrained recovery, the augmentation (2.30) should be conducted for all $I$ in this case.

\section{Linearization of the mixed formulation}

The algorithm for the treatment of normal contact with a constrained recovery is summarized in Table 1 . Within a Newton-Raphson approach, the variational term $\delta \mathcal{G}_{N}^{c}$ contributes to the force vector whereas $-\Delta \delta \mathcal{G}_{N}^{c}$ contributes to the tangent matrix:

$-\Delta \delta \mathcal{G}_{N}^{c}=\int_{\partial \mathcal{R}_{0}^{c}}\left(\Delta \delta g_{N} p_{N}+\delta g_{N} \Delta p_{N}\right) \mathrm{d} A$.
Here, $\Delta \delta g_{N}$ is delivered by the continuum formulation of contact and is a standard term - see $[23,47]$. It is recalled that this yields a symmetric form. Note that it is not necessary to consider the linearization (or, variation) of the Jacobian of isoparametric mapping in the present context since all integration is carried out in the reference configuration - see [41] for the choice of the current configuration. Using (2.17) together with the standard expression for $\delta g_{N}$, the second term can be expressed as

$\int_{\partial \mathcal{R}_{o}^{c}} \delta g_{N} \Delta p_{N} \mathrm{~d} A=-\sum_{I / J} \sum_{K} \int_{\partial \mathcal{R}_{o}^{c}}\left(\delta x_{i}^{I} N^{I}-\delta y_{i}^{J} M^{J}\right) v_{i} \in{ }_{N} \chi^{K} N^{K} \Delta \gamma_{N}^{K} \mathrm{~d} A$.

Introducing normal-weighted overlap integrals

$\stackrel{x^{I J}}{\Omega_{i}^{I J}}:=\int_{\partial \mathcal{R}_{0}^{c}} N^{I} v_{i} N^{J} \chi^{J} \mathrm{~d} A$ and $\stackrel{{ }^{y}}{\Omega_{i}^{I J}}:=\int_{\partial \mathcal{R}_{0}^{c}} N^{I} v_{i} M^{J} \chi^{J} \mathrm{~d} A$,

which only need to be evaluated for $\chi^{J}=1$, one obtains

$\int_{\partial \mathcal{R}_{0}^{c}} \delta g_{N} \Delta p_{N} \mathrm{~d} A=-\epsilon_{N} \sum_{I / J} \sum_{K}\left(\delta x_{i}^{I} \Omega_{i}^{K I}-\delta y_{i}^{J} \Omega_{i}^{K^{K J}}\right) \chi^{K} \Delta \gamma_{N}^{K}$.

The normal-weighted overlap integrals depend on the contact status via $v$ and therefore must be reevaluated at each iteration. However, this does not cause a significant additional computational cost. The active set indicator $\chi^{K}$ is retained explicitly in the integrals to highlight that several sums are typically conducted efficiently over a small subset of the contact degrees of freedom. These integrals are classically avoided by defining a suitable discrete normal field $[33,10]$ so that evaluating the mortar overlap integrals once in a pre-processing stage is sufficient.

The linearization $\Delta \gamma_{N}^{K}$ follows from Eq. (2.23):

$\Delta \gamma_{N}^{K}=\sum_{L} \widehat{\Psi}^{K L} \chi^{L} \Delta \bar{g}_{N}^{L}$

Employing

$$
\begin{aligned}
\Delta \bar{g}_{N}^{L} & =\left\langle N^{L} \Delta g_{N}\right\rangle=-\sum_{P / Q}\left\langle N^{L}\left(N^{P} \Delta x_{j}^{P}-M^{Q} \Delta y_{j}^{Q}\right) v_{j}\right\rangle \\
& =-\sum_{P / Q}\left(\stackrel{x}{\Omega}_{j}^{L P} \Delta x_{j}^{P}-\stackrel{y}{\Omega}_{j}^{L Q} \Delta y_{j}^{Q}\right)
\end{aligned}
$$

one obtains the final expression

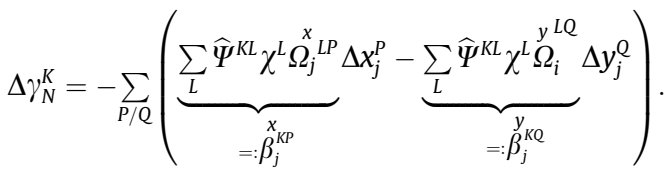

It is remarked that ${ }^{x / y}{ }_{j}^{A B}$ is not identity with respect to the indeces $A B$, in general. In order to employ the lumped treatment of Section 2.5.2, $\widehat{\Psi}^{I I}$ is replaced by $\delta_{I J} / \psi^{I}$ where $\delta_{I J}$ is the Kronecker delta. For the unconstrained treatment of Section 2.5.1, $\widehat{\Psi}^{K L}$ is replaced by $\widehat{\Phi}^{K L}$ and $\chi^{K}$ is removed from (3.2) as well as from all terms emanating from it.

Using these results for the constrained formulation, one obtains the linearization

$$
\int_{\partial \mathcal{R}_{o}^{c}} \delta g_{N} \Delta p_{N} \mathrm{~d} A=\epsilon_{N} \sum_{I / J} \sum_{K} \sum_{P / Q}\left(\delta x_{i}^{I} \stackrel{x}{\Omega}_{i}^{K I}-\delta y_{i}^{J} \stackrel{y}{\Omega}_{i}^{K J}\right) \chi^{K}\left(\stackrel{x}{\beta_{j}^{K P}} \Delta x_{j}^{P}-{\stackrel{y}{\beta_{j}^{K Q}}}^{K Q} y_{j}^{Q}\right)
$$

which defines the tangent matrix entries $K_{i j}^{A B}$ that relate the variations $\left\{\delta x_{i}^{A}, \delta y_{i}^{A}\right\}$ to the increments $\left\{\Delta x_{j}^{B}, \Delta y_{j}^{B}\right\}$.

This result, in view of the definitions in (3.7), displays the symmetry of the normal contact formulation. This symmetry is not present in all penalty-regularized mortar formulations. For instance, in [33] it was regarded to be of secondary importance compared to computational ease of implementation. 
Table 1

Algorithm for normal contact treatment with Uzawa augmentation and constrained recovery.

1. Initialize. Evaluate the gap $g_{N}$ at the closest-point projection. The mixed pressure $p_{N}^{I, n}=p_{N}^{I,(0)}$ at time/load step $n$ is known. Within a Newton-Raphson iteration of the $k$-th Uzawa augmentation at time/load step $n+1$, compute the augmentation pressure and the overlap integrals:

$p_{N}^{(k)}=\sum_{I} N^{I} p_{N}^{I,(k)}, \Phi^{I J}=\left\langle N^{I} N^{J}\right\rangle$

2. Projected mortar variables. Compute projections of kinematic and kinetic variables:

$\bar{g}_{N}^{I}=\left\langle N^{I} g_{N}\right\rangle, \bar{p}_{N}^{I,(k)}=\left\langle N^{I} p_{N}^{(k)}\right\rangle$

3. Active-set update. Check new contact and loss of contact to determine $\mathcal{A}=\left\{I \mid \chi^{I}=1\right\}$ :

$\chi^{I}=\left\{\begin{array}{l}0 \\ 1\end{array}\right\}$ and $\left\{\begin{array}{l}\bar{g}_{N}^{I}>0 \\ \bar{p}_{N}^{I}<0\end{array}\right\}$ : contact is $\left\{\begin{array}{c}\text { initiated } \\ \text { lost }\end{array}\right\} \rightarrow \chi^{I}=\left\{\begin{array}{l}1 \\ 0\end{array}\right\}$

4. Mixed kinematic variable recovery. Compute the nodal values of the mixed kinematic variable:

$\widehat{\Psi}^{I J}=\chi^{I} \Phi^{I J} \chi^{J} \rightarrow \gamma_{N}^{I}=\left\{\begin{array}{ll}0 & \text { if } \chi^{I}=0 \\ \sum_{J} \widehat{\Psi}^{I J} \chi^{J} \bar{g}_{N}^{J} & \text { if } \chi^{I}=1\end{array}\right.$.

5. Mixed kinetic variable update. Update the mixed, and optionally the projected, pressure at $I \in \mathcal{A}$ :

$p_{N}^{I}=p_{N}^{I,(k)}+\epsilon_{N} \gamma_{N}^{I} \leftrightarrow \bar{p}_{N}^{I}=\bar{p}_{N}^{I,(k)}+\epsilon_{N} \bar{g}_{N}^{I}$

6. Local traction. Compute the updated local traction for the weak form evaluation and solution:

$p_{N}=\sum_{I} N^{I} p_{N}^{I} \rightarrow \delta \mathcal{G}_{N}^{c}=-\int_{\partial \mathcal{R}_{o}^{c}} \delta g_{N} p_{N} \mathrm{~d} A$

7. Check convergence. Estimate the error in augmentation iteration $k+1$ and reiterate unless

$\mathfrak{£}^{(k+1)}<$ Tol

\section{Numerical investigations}

In this section, major aspects of the three types of mixed formulation for frictionless mortar-based contact are investigated within a quasistatic setting. The local solution quality is investigated by monitoring the pressure distribution, in Section 4.1 for the classical Hertzian contact with two deformable bodies and in Section 4.2 for a Hertzian-type contact with a rigid surface in the finite deformation regime. In Section 4.3, the classical patch test with a flat frictionless contact interface is conducted. The global solution quality and robustness of the three variants of the mixed formulation are assessed in Section 4.4 through an indentation problem by monitoring the contact forces and in Section 4.5 through the large sliding contact of two thick-walled shells.

For the volumetric formulation, a classical Neo-Hookean type material model will be employed based on the strain energy function $\left(J=[\operatorname{det}] \boldsymbol{F}\right.$ and $\left.\boldsymbol{C}=\boldsymbol{F}^{T} \boldsymbol{F}\right)$

$\mathcal{W}=\frac{\Lambda_{1}}{2}(\ln J)^{2}+\frac{\Lambda_{2}}{2}\left(J^{-2 / 3} \operatorname{tr}[\boldsymbol{C}]-3\right)$.

Here, the bulk and shear moduli $\left\{\Lambda_{1}, \Lambda_{2}\right\}$ correspond to the choices of a Young's modulus $E=10$ and a Poisson's ratio $v=0.3$. Only for the Hertzian contact problem, $E=1$ is employed. In all examples, the volume is discretized with either linear Lagrange or quadratic NURBS elements, the latter based on the recent developments in $[40,26,5]$. The volume integrals are evaluated with two or four integrations points, respectively, per parametric direction of an element. Throughout the developments, the terminology node was employed in the context of discretization, although control point would be more appropriate for NURBS which are also non-interpolatory in general. The classical choice has been retained for simplicity.

The contact discretization is directly inherited from the volume parametrization as its specialization to the surface. Within the contact formulation, $\epsilon_{N}$ will denote the base value of the penalty parameter. Before being transferred to the contact computations, this base value is multiplied by the largest diagonal entry of the bulk stiffness matrix associated with the deformable bodies at the first Newton-Raphson iteration of the first Uzawa augmentation, but subsequently kept constant throughout the load step. In order to assess the convergence of the Uzawa iterations based on the criterion (2.32), the error measure $\mathfrak{f}^{(k+1)}:=\frac{\left\|\prod^{(k+1)}-\prod^{(k)}\right\|_{2}}{\left\|\prod^{(k)}\right\|_{2}}$

is introduced where $\prod^{(k)}$ denotes the vector of all augmentation pressures $p_{N}^{I,(k)}$. A convergence tolerance of ToL $=0.01$ was found to deliver very accurate results in a variety of examples while $\mathrm{ToL}=0.001$ ensures a converged pressure distribution for the chosen base values of $\epsilon_{N}$. Values of $\epsilon_{N}$ and ToL will be denoted explicitly for each example together with the number of integration points employed for the evaluation of the contact integrals. In all of the figures presented, the upper (blue) ${ }^{2}$ body is chosen as the slave and the lower (yellow) one as the master. In the classical Hertz contact example, the roles of master and slave will additionally be switched.

For all comparisons, the abbreviations Con (constrained), LMP (lumped) and UNC (unconstrained) are used to refer to the recovery method for the mixed variables. All figures displaying mesh deformation were computed with constrained recovery. Finally, load step adaptivity was used in all computations to automatically circumvent convergence problems with large load steps although adaptation was not necessary in most examples.

\subsection{Classical Hertzian contact}

The classical Hertzian contact problem is considered within a plane-strain setting with two deformable bodies. The problem setup is summarized in Fig. 1. Here, the upper body is displaced downwards in ten load steps by prescribing the nodal positions on the upper and side surfaces while the lower body is held fixed via the same set of nodes. $\epsilon_{N}=10$ and $\mathrm{ToL}=0.001$ are chosen together with non-matching discretizations. More specifically, 48 linear Lagrange elements are used in the radial direction for both bodies. Along the angular direction, three levels of NURBS discretizations are used for the upper/lower body with the number of elements equal to (i) 48 / 24 for the coarse, (ii) $72 / 48$ for the intermediate, and (iii) 96/72 the fine resolution. The elements are concentrated to the vicinity of the contact surface for numerical efficiency. To ensure an accurate eval-

\footnotetext{
${ }^{2}$ For interpretation of colour in Figs. 1-7 and 9-11, the reader is referred to the web version of this article.
} 


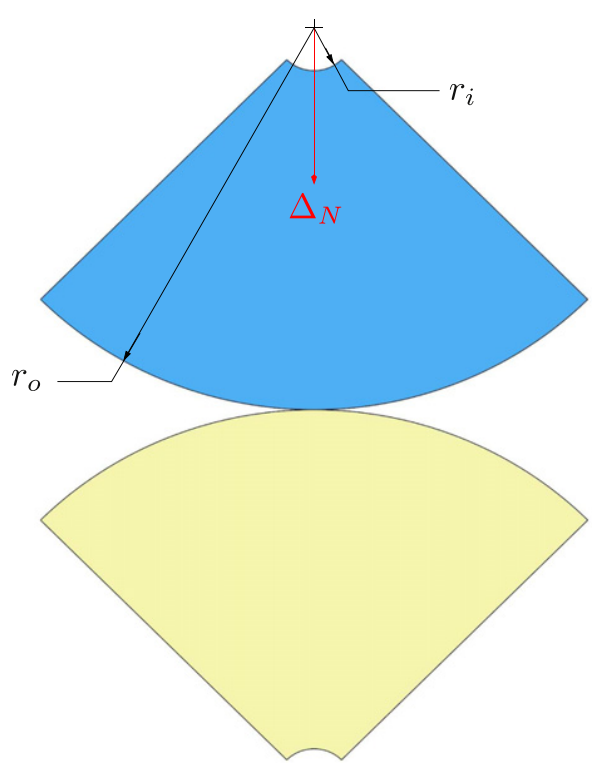

(a) problem geometry

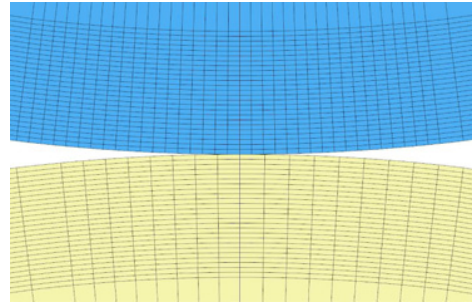

(b) undeformed interface

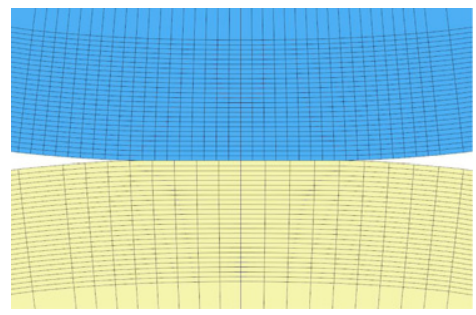

(c) deformed interface

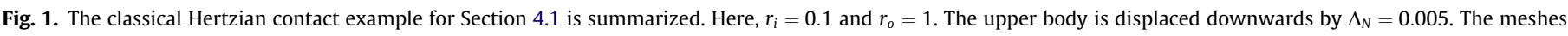
shown correspond to the coarse resolution.

uation of the contact integrals, 30 integration points are employed. Since $N^{I} \geqslant 0$ is ensured for NURBS, $\psi^{I}>0$ and hence $p_{N} \geqslant 0$ is guaranteed for the lumped recovery approach.

Comparisons of the three recovery approaches are summarized in Fig. 2. Due to the underlying formulation, the support of the pressure distribution remains localized for unconstrained and lumped recovery, both delivering similar results which overlap in some instances. For the unconstrained approach, a non-zero pressure is observed well beyond the analytical contact zone, accompanied by decaying oscillations. Such oscillations are observed in early mortar-based methods as well [13] and are strongly influenced by elements that span the edge of the contact zone from the analytical contact region into the non-contact one [21,9]. Although the rate of convergence is not monitored in this work, for all recovery approaches the oscillations diminish in magnitude with increasing mesh resolution, indicating local convergence. Still, however, the stability of unconstrained recovery may be called into question due to the checkerboard mode type pressure distribution that it delivers (cf. [38]), which is also observed in Section 4.2. As in the classical NTS approach, the implication would be that the oscillations may not necessarily diminish further with further increases in the mesh resolution [6,38]. However, these oscillations occur predominantly in low pressure regions where contact is not expected theoretically (see also Section 4.2) and the method delivers an improvement to the global solution quality as will be observed in Section 4.5 in addition to passing the patch test (Section 4.3) as well as not displaying locking (Section 4.2). Consequently, it is retained as a convenient alternative without pursuing an explicit stability analysis.

Since the present mortar-based approach discretizes the mixed variables on the slave surface only, the designation of the master and slave influences the results which introduces a geometric bias as in the classical one-pass node-to-segment approaches [29]. While such a geometric bias may be undesirable in particular for cases where the master/slave designation may not be automatically assigned a priori $[8,38]$, it is only prominent at coarse discretizations and diminishes with mesh resolution as shown in Fig. 2. Therefore, this effect will not be additionally monitored in the remaining exam- ples of this work. Finally, Fig. 3 displays the identical convergence behavior of the three methods based on the $L_{2}$-norm of the error in the normalized pressure distribution. Here, mesh refinement is driven by a parameter $n$ such that the bodies are discretized with $2 n$ NURBS elements in the radial direction while the slave/master is discretized with $16 n / 4 n$ NURBS elements in the angular direction. For the computation of the analytical pressure distribution, the load corresponding to the imposed displacement is determined using $n=32$.

\subsection{Finite Hertzian-Type contact}

The finite deformation Hertzian-type contact problem is considered with a rigid surface. The problem setup is summarized in Fig. 4 with representative dimensions where the deformable body is displaced downwards by prescribing the nodal positions on the upper (square) surface. $\epsilon_{N}=1000$ and $\mathrm{ToL}=0.001$ are chosen. For the evaluation of the contact integrals, six integration points are employed.

The pressure distributions corresponding to different recovery methods are presented in Fig. 5 for three different discretizations with the number of Lagrange elements along the horizontal/vertical direction equal to (i) $12 / 6$ for the coarse, (ii) $18 / 9$ for the intermediate, and (iii) $24 / 12$ the fine resolution. Here, although not precluded by the theory, negative pressure zones for constrained and unconstrained recovery are highlighted. Since $N^{I} \geqslant 0$ is ensured for linear Lagrange elements, $\psi^{I}>0$ and hence $p_{N} \geqslant 0$ is again guaranteed for the lumped recovery approach. The pressure distributions are already identical at the intermediate resolution. Moreover, at this resolution, the pressure magnitude for unconstrained recovery away from the contact zone predicted by lumped recovery is negligible in magnitude. Constrained recovery can then be interpreted as a consistent method of imposing a cut-off criterion whereby these negligible pressures are omitted. However, this consistency is meaningful at sufficiently high resolutions. Clearly, such a cut-off is not meaningful for the coarse resolution result. It is remarked that the high quality of the solutions with Uzawa augmentations also confirms that the present class of algorithms does not display surface locking $[38,40]$. 

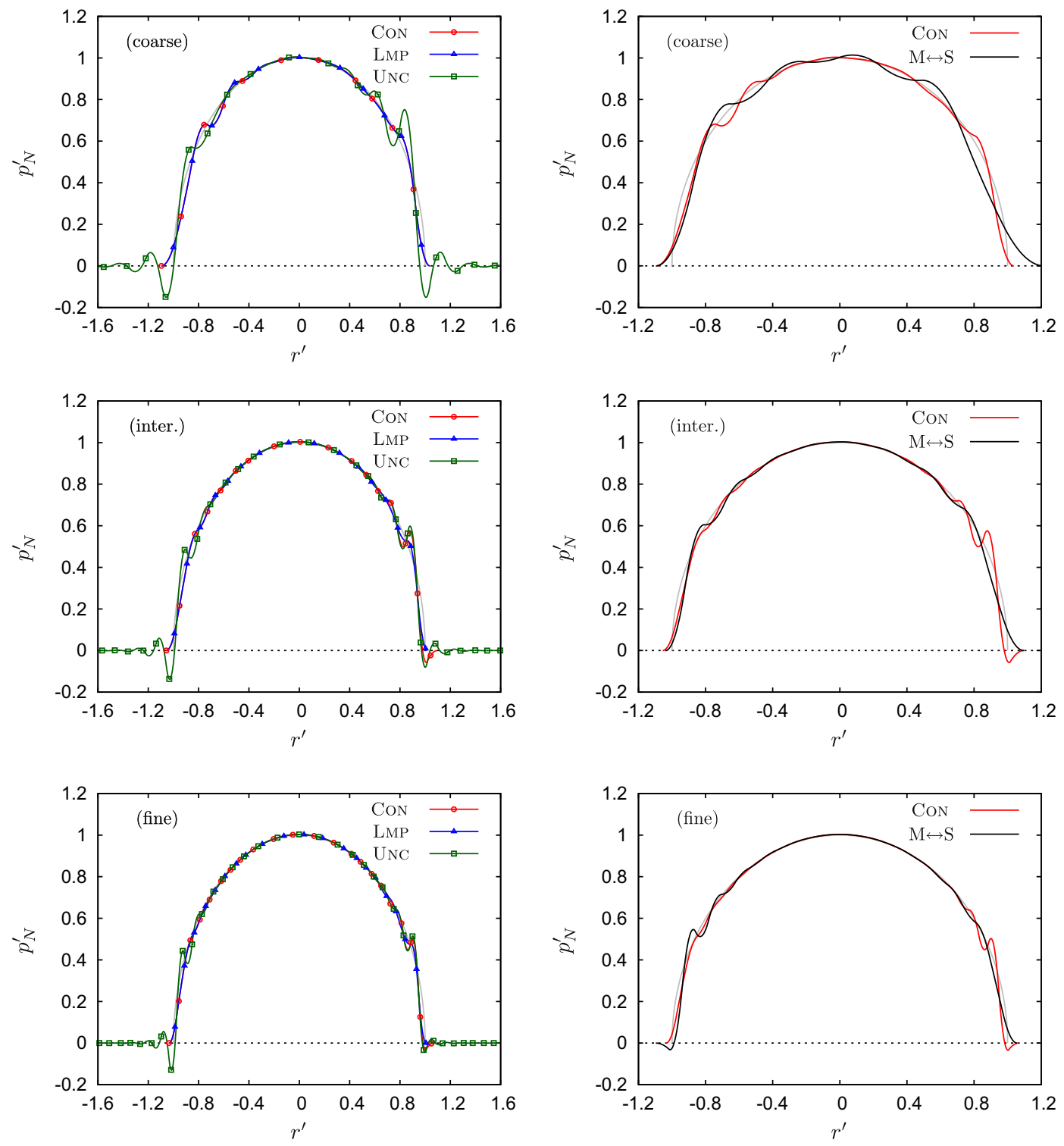

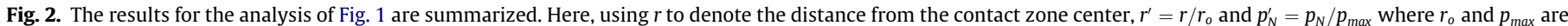

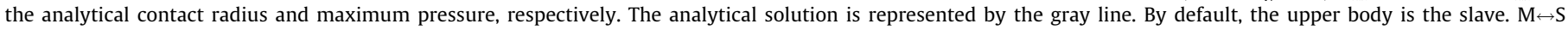
indicates switching master and slave choices.
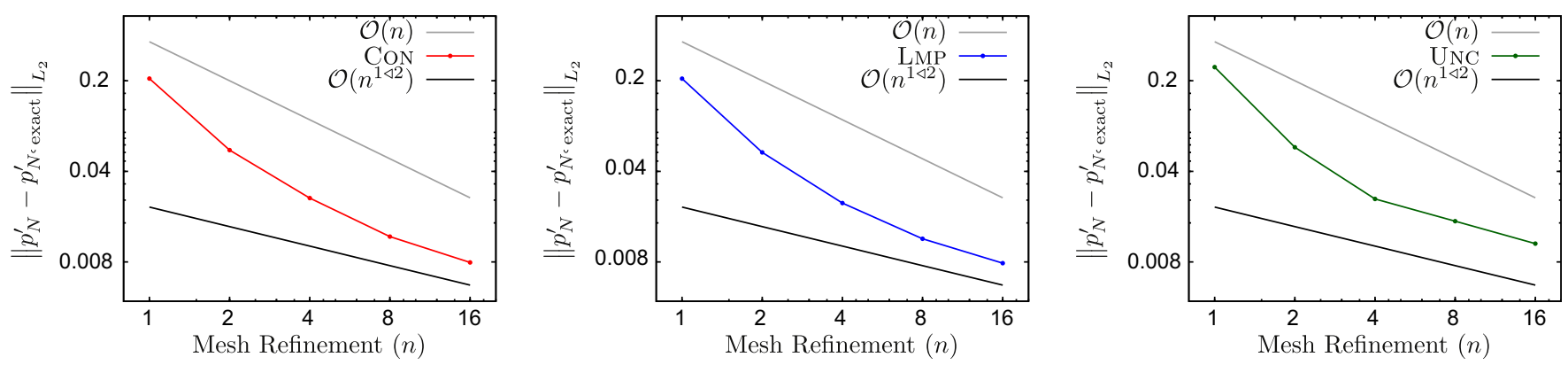

Fig. 3. $L_{2}$-norm of the error in the normalized pressure distribution is monitored for the Hertzian contact problem of Section 4.1 .

\subsection{Patch test: flat interface}

Within the present framework, the finite element discretization of the pressure, in a manner that is consistent with the volume discretization, ensures that the patch test will be satisfied. However, this additionally requires an exact evaluation of the contact inte- grals - only possible for flat interfaces - which is typically realized through the segmentation of the contact interface. Presently, segmentation is not pursued. Consequently, the integrals are evaluated approximately using 20 integration points per interface direction - see [7] for studies of quadrature on the slave surface. 


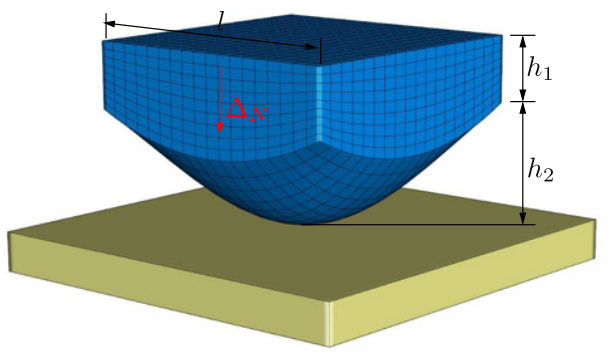

(a) problem geometry

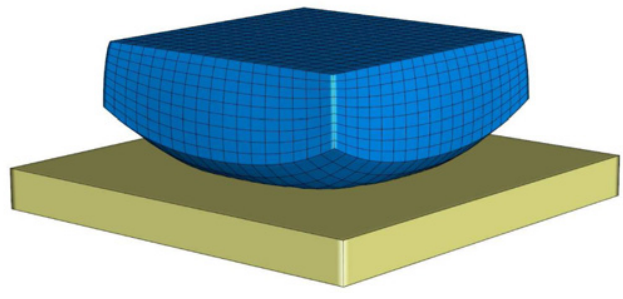

(b) deformed configuration

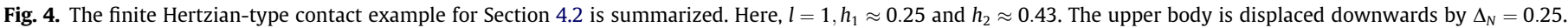
The initial gap between the surfaces is approximately 0.01 .

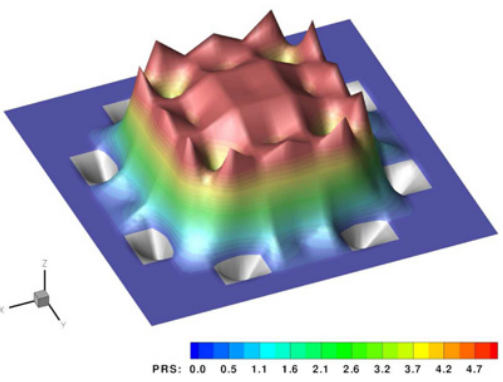

(a-1) CoN (coarse)

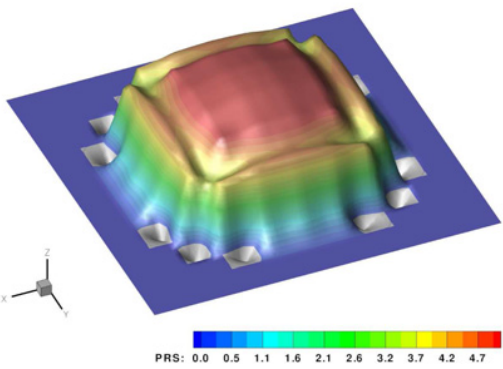

(b-1) CoN (inter.)

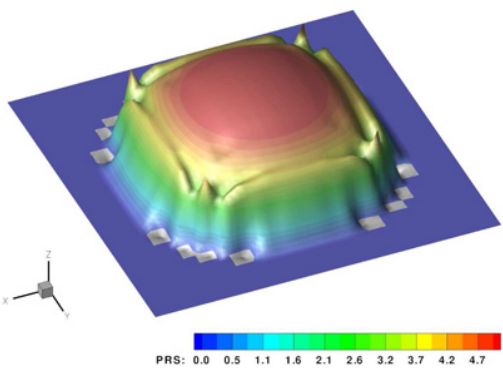

(c-1) Con (fine)

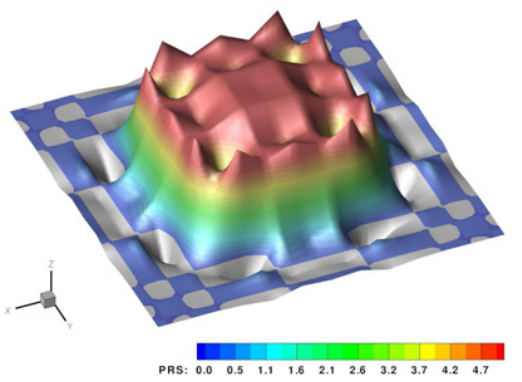

(a-2) UNC (coarse)

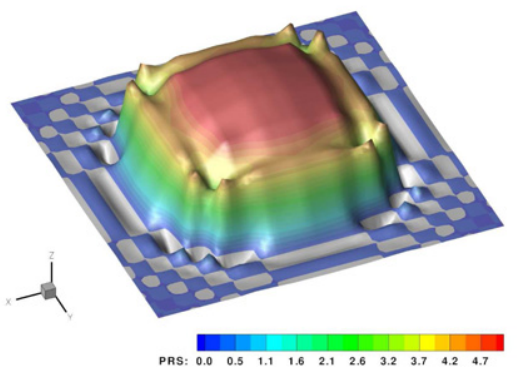

(b-2) UNC (inter.)

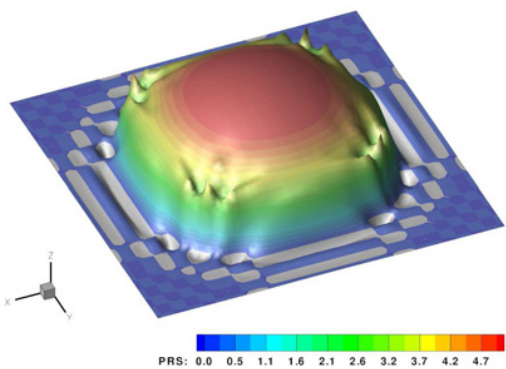

(c-2) UNC (fine)

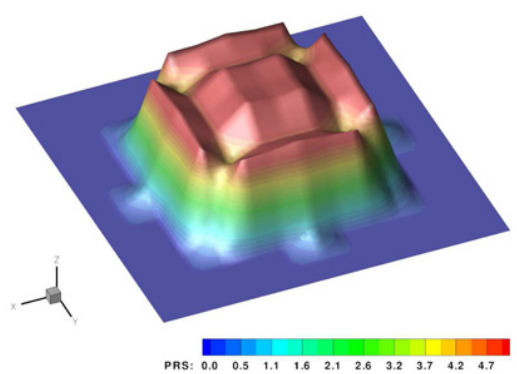

(a-3) LMP (coarse)

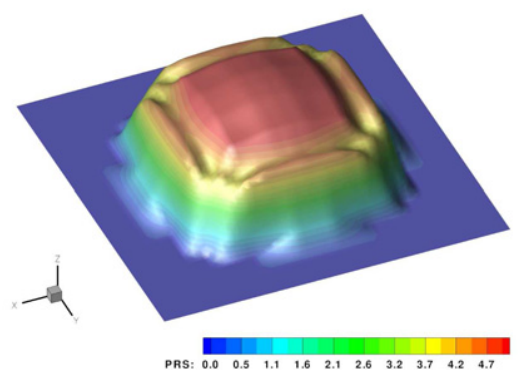

(b-3) LMP (inter.)

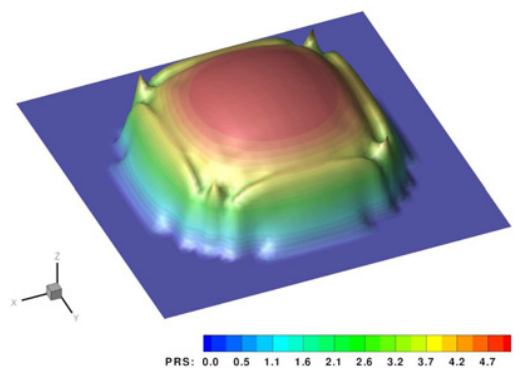

(c-3) LMP (fine)

Fig. 5. The pressure $\left(\mathrm{PRS}=p_{N}\right)$ distributions for the analysis of Fig. 4 are shown. Negative pressure zones are are highlighted by gray shading.

The geometry for the patch test is shown in Fig. 6, where the only interface constraints are the Hertz-Signorini-Moreau conditions. Here, $\epsilon_{N}=100$ and $\mathrm{ToL}=0.001$ For other patch test setups, see $[29,4,6]$. While the meshes are non-matching at this minimal Lagrange discretization, the bodies are expected to deform as one. Qualitatively, this is satisfied. Quantitatively, the interface pressure is not exactly a constant due to the approximate evaluation of the contact integrals. However, the oscillations are negligible in magnitude and the stress distribution throughout the bodies remains approximately constant as well. Here, unconstrained recovery has been employed, which is presently equivalent to constrained recovery since the complete interface is in contact. The results are identical for lumped recovery.

\subsection{Indentation of an elastic block}

Fig. 7 summarizes the indentation of an elastic block $(E=1)$ by a stiffer body $(E=100)$ such that at the end of the loading the upper body is completely embedded in the lower one. In this case, the high stiffness ratio renders the computations challenging. Here, $\epsilon_{N}=100$ and ToL $=0.01$ are employed with two NURBS discretizations. For the coarse resolution, the upper body is discretized with 


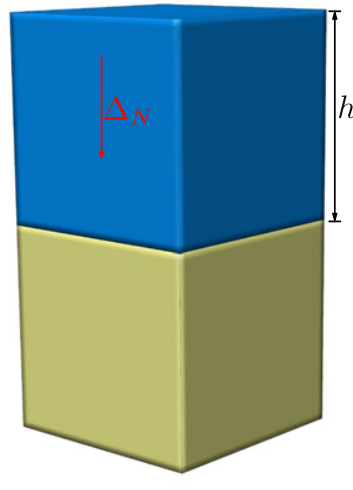

(a) problem geometry

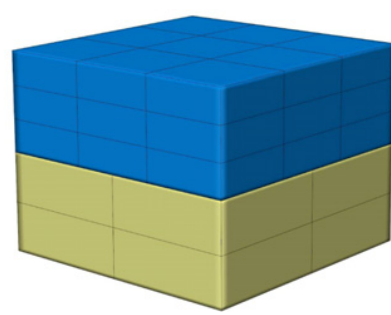

(b) deformed configuration

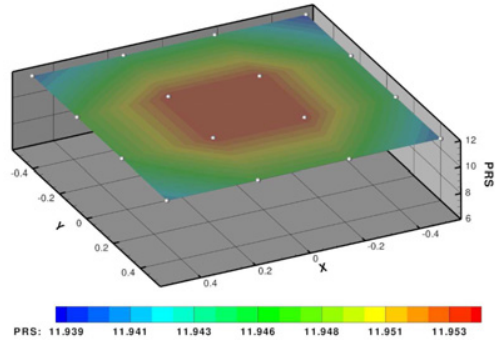

(c) interface pressure

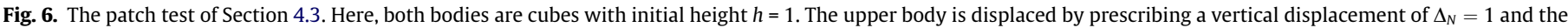

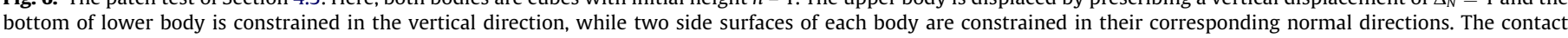
pressure $\left(\operatorname{PRS}=p_{N}\right)$ is constant to within quadrature error.

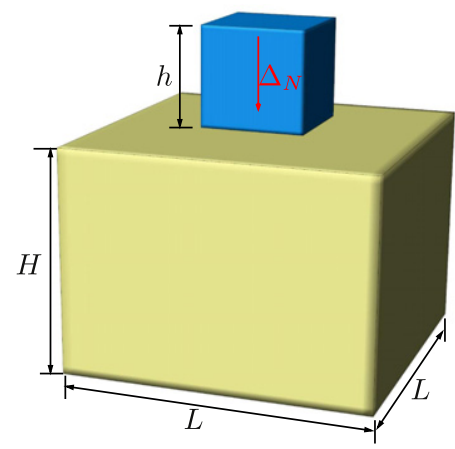

(a) problem geometry

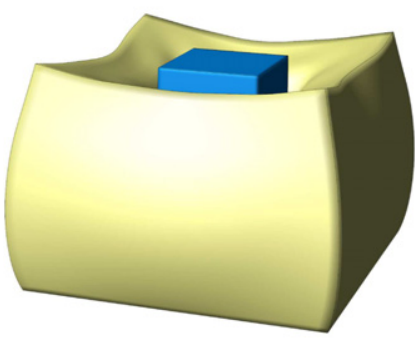

(b) deformed configuration (side)

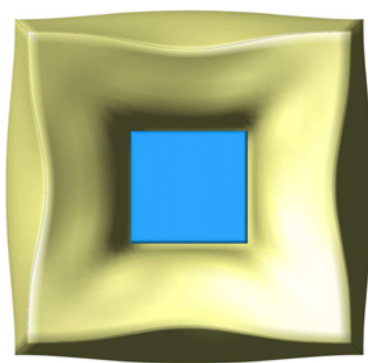

(c) deformed configuration (top)

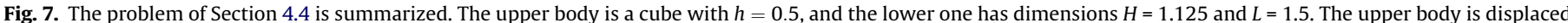

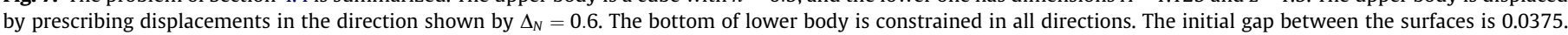
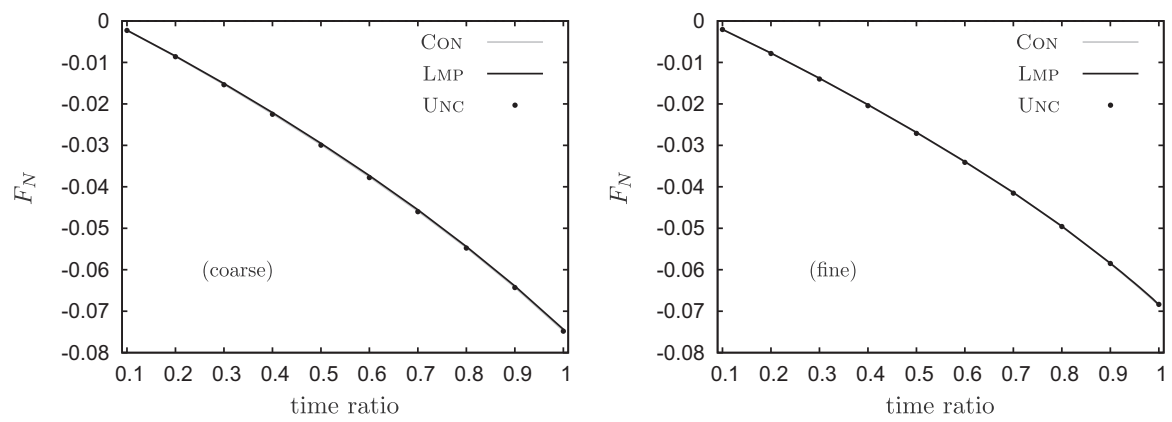

Fig. 8. The normal forces $\left(F_{N}\right)$ monitored in the analysis of Fig. 7 are summarized.
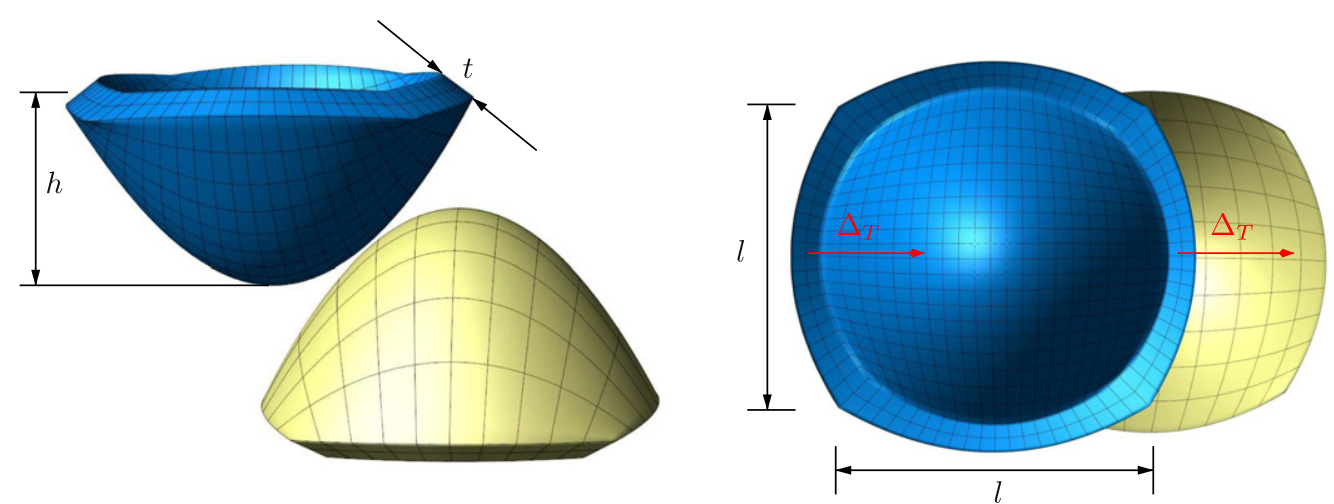

Fig. 9. The problem of Section 4.5 is summarized. Here, $t \approx 0.12, l \approx 1$ and $h \approx 0.68$. The upper body is tangentially displaced by $\Delta_{T}=1.2$ while the lower one is fixed. 


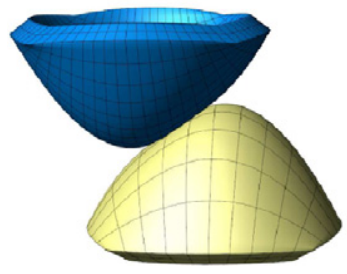

(a) time ratio $=0.2$

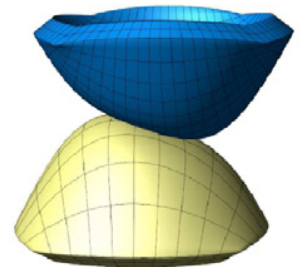

(d) time ratio $=0.6$

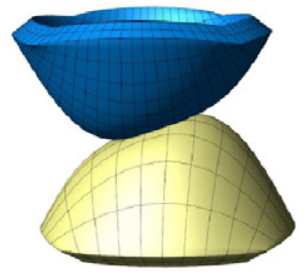

(b) time ratio $=0.4$

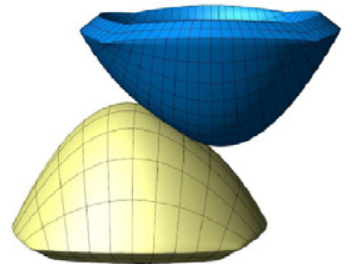

(e) time ratio $=0.8$

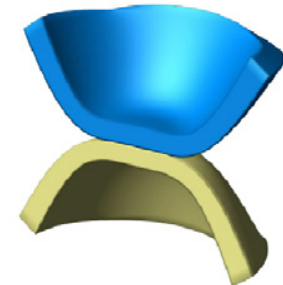

(c) time ratio $=0.5$

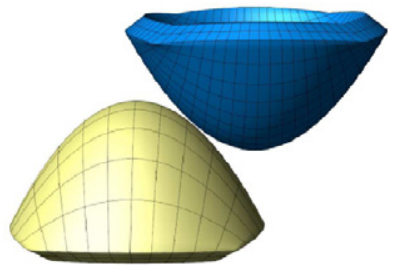

(f) time ratio $=1.0$

Fig. 10. Simulation instances from the problem of Fig. 9 at the intermediate resolution.
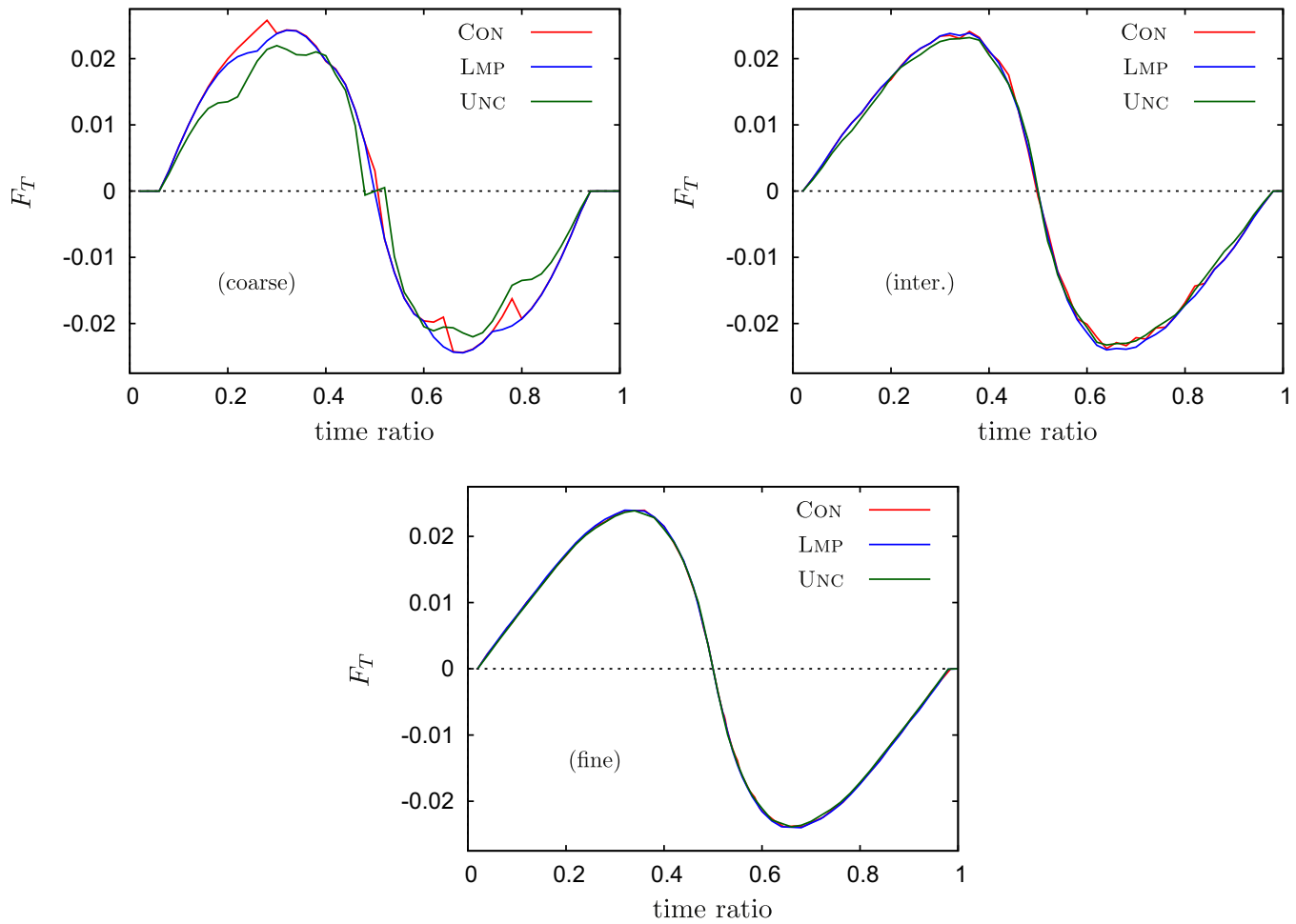

Fig. 11. The tangential forces $\left(F_{T}\right)$ monitored the analysis of Fig. 9 are summarized.

4/2 elements per horizontal/vertical direction while the combination for the lower one is $5 / 3$. For the fine resolution, an identical resolution combination of $8 / 4$ is employed. As global indicators of the contact interactions, the measured normal forces applied on the upper body for the three types of recovery approaches are shown in Fig. 8. Clearly, while the local pressure distributions are generally different for the three methods, they deliver quantitatively very similar results even at coarse resolutions under such large deformations. However, in the next section, it is demonstrated that this observation does not hold in general.

\subsection{Thick-walled shells in sliding contact}

Fig. 9 depicts the large sliding problem for two thick-walled shells together with representative dimensions. Here, the upper body is displaced tangentially in 50 load steps by prescribing dis- placements at the leading and trailing edges of the top surface. The lower body is held fixed at the corresponding edges. The initial and final configurations are symmetric with respect to the center of the lower body. $\epsilon_{N}=10$ and ToL $=0.01$ are chosen and the thickness directions are resolved by two NURBS elements. The lower body is kept at a constant resolution of 10 elements per tangential direction while the upper body is discretized at three levels: (i) 10 for coarse, (ii) 20 for intermediate and (iii) 30 for fine resolutions. The corresponding number of contact element integration points per direction is 12,8 and 6 , respectively.

Instances from the simulation are shown in Fig. 10. The twodimensional version of this problem with two circular arches displays a snap-through instability among the two arches (versus within one arch) even at mild interactions and therefore requires either a dynamic simulation [31] or an improved Newton-Raphson scheme for a quasistatic solution [48]. Its present three- 
dimensional counterpart, however, does not physically display a snap-through for the given setup.

The contact interactions are monitored at the global level through the tangential force applied to the upper body as summarized in Fig. 11 for the three types of recovery approaches. At the fine resolution, the response curves from all methods overlap. For all resolutions, lumped recovery delivers a smooth and symmetric force distribution as is expected from this problem. This expectation highlights a problem with constrained recovery. Symmetry is lost and jumps in the measured forces are clearly observed even at the intermediate resolution. The source of this inconsistency is the earlier mentioned non-smooth relationship between the mixed and projected kinematic variables. This highlights the advantage of using unconstrained recovery, for which the force distributions are smooth and symmetric although the local pressure distributions are more oscillatory compared to the other recovery approaches at these levels of discretization. It is remarked that the single jump for the coarse resolution of unconstrained recovery, where only a few nodes are active, is due to a non-physical mild snap-through within the upper shell. This phenomenon already disappears at the intermediate resolution.

\section{Conclusion}

In this work, the relationship between a classical three-field mixed variational formulation of frictionless contact mechanics and recent mortar-based treatments was investigated. Within a mixed variational framework, the mortar projection operators appear naturally. Three different recovery approaches were investigated to obtain the mixed kinematic and kinetic quantities from their projected counterparts: (i) constrained, (ii) lumped and (iii) unconstrained. The close relationship between these approaches and earlier mortar implementations from the mathematical and engineering literatures was additionally highlighted, hence providing a convenient framework for the derivation of a mortarbased approach. In particular, while the lumped approach appears to be computationally the most appealing, its formulation is based on the unconstrained approach that delivers the constrained one as a special case as well. For a robust numerical performance, augmentation in the context of Uzawa iterations was discussed and the linearization of the overall algorithm was presented. Two- and three-dimensional numerical investigations in the infinitesimal and finite deformation regimes assessed the performance of these mortar-based contact treatment approaches with respect to local pressure distributions as well as via global force monitoring.

The present study lays the foundations for an identical derivation of a class of mortar-based frictional contact algorithms. The ability to incorporate frictional contact will additionally allow, by restricting slip, a convenient handling of mesh tying constraints that are needed in domain decomposition techniques. Consequently, it will be possible to assess the performance of the proposed class of contact algorithms through patch tests with curved interfaces. Such studies are currently being pursued by the author.

\section{Acknowledgements}

Support for this work was provided by the Scientific and Technological Research Council of Turkey (TÜBITAK) under the Career Programme Grant No. 110M661.

\section{References}

[1] P. Alart, A. Curnier, A mixed formulation for frictional contact problems prone to Newton like solution methods, Comput. Methods Appl. Mech. Engrg. 92 (1991) 353-375.
[2] F. Ben Belgacem, P. Hild, P. Laborde, Approximation of the unilateral contact problem by the mortar finite element method, C. R. Acad. Sci. Paris. Série I 324 (1997) 123-127.

[3] T. Cichosz, M. Bischoff, Consistent treatment of boundaries with mortar contact formulations using dual Lagrange multipliers, Comput. Methods Appl. Mech. Engrg. 200 (2011) 1317-1332.

[4] M.A. Crisfield, Re-visiting the contact patch test, Int. J. Numer. Methods Engrg 48 (2000) 435-449.

[5] L. De Lorenzis, I. Temizer, P. Wriggers, G. Zavarise, A large deformation frictional contact formulation using NURBS-based isogeometric analysis, Int. J. Numer. Methods Engrg. 87 (2011) 1278-1300.

[6] N. El-Abbasi, K.J. Bathe, Stability and patch test performance of contact discretizations and a new solution algorithm, Comput. Struct. 79 (2001) 1473 1486 .

[7] K.A. Fischer, P. Wriggers, Frictionless 2D contact formulations for finite deformations based on the mortar method, Comput. Mech. 36 (2005) 226-244

[8] B. Flemisch, M.A. Puso, B.I. Wohlmuth, A new dual mortar method for curved interfaces: 2D elasticity, Int. J. Numer. Methods Engrg. 63 (2005) 813-832.

[9] D. Franke, A. Düster, V. Nübel, E. Rank, A comparison of the h-, p-, hp-, and rpversion of the FEM for the solution of the 2D Hertzian contact problem, Comput. Mech. 45 (2010) 513-522.

[10] C. Hesch, P. Betsch, A mortar method for energy-momentum conserving schemes in frictionless dynamic contact problems, Int. J. Numer. Methods Engrg. 77 (2009) 1468-1500.

[11] C. Hesch, P. Betsch, Transient three-dimensional domain decomposition problems: frame-indifferent mortar constraints and conserving integration, Int. J. Numer. Methods Engrg. 82 (2010) 329-358.

[12] C. Hesch, P. Betsch, Transient three-dimensional contact problems: mortar method. mixed methods and conserving algorithms, Comput. Mech. 48 (2011) 461-475.

[13] P. Hild, Numerical implementation of two nonconforming finite element methods for unilateral contact, Comput. Methods Appl. Mech. Engrg. 184 (2000) 99-123.

[14] P. Hild, P. Laborde, Quadratic finite element methods for unilateral contact problems, Appl. Numer. Anal. 41 (2002) 401-421.

[15] P. Hild, Y. Renard, A stabilized Lagrange multiplier method for the finite element approximation of contact problems in elastostatics, Numer. Math. 115 (2010) 101-129.

[16] M. Hintermüller, K. Ito, K. Kunisch, The primal-dual active set strategy as a semismooth Newton method, SIAM J. Optim. 13 (2003) 865-888.

[17] S. Hüeber, M. Mair, B.I. Wohlmuth, A priori error estimates and an inexact primal-dual active set strategy for linear and quadratic finite elements applied to multibody contact problems, Appl. Numer. Math. 54 (2005) 555-576.

[18] S. Hüeber, B.I. Wohlmuth, A primal-dual active set strategy for non-linear multibody contact problems, Comput. Methods Appl. Mech. Engrg. 194 (2005) 3147-3166.

[19] S. Hüeber, B.I. Wohlmuth, Thermo-mechanical contact problems on nonmatching meshes, Comput. Methods Appl. Mech. Engrg. 198 (2009) 13381350.

[20] R.E. Jones, P. Papadopoulos, A novel three-dimensional contact finite element based on smooth pressure interpolations, Int. J. Numer. Methods Engrg. 51 (2001) 791-811.

[21] A. Konyukhov, K. Schweizerhof, Incorporation of contact for high-order finite elements in covariant form, Comput. Methods Appl. Mech. Engrg. 198 (2009) 1213-1223

[22] A. Konyukhov, K. Schweizerhof, Geometrically exact theory for contact interactions of 1D manifolds. Algorithmic implementation with various finite element models, Comput. Methods Appl. Mech. Engrg. 205-208 (2012) 130138 .

[23] T.A. Laursen, Computational Contact and Impact Mechanics, first ed., Springer Berlin Heidelberg New York, 2003 (corr. 2nd printing).

[24] T.A. Laursen, M.A. Puso, J. Sanders, Mortar contact formulations for deformable-deformable contact: past contributions and new extensions for enriched and embedded interface formulations, Comput. Methods Appl. Mech. Engrg. 205-208 (2012) 3-15

[25] F. Liu, R.I. Borja, Stabilized low-order finite elements for frictional contact with the extended finite element method, Comput. Methods Appl. Mech. Engrg. 199 (2010) 2456-2471.

[26] J. Lu, Isogeometric contact analysis: geometric basis and formulation of frictionless contact, Comput. Methods Appl. Mech. Engrg. 200 (2011) 726-741.

[27] T.W. McDevitt, T.A. Laursen, A mortar-finite element formulation for frictiona contact problems, Int. J. Numer. Methods Engrg. 48 (2000) 1525-1547.

[28] P. Papadopoulos, J.M. Solberg, A Lagrange multiplier method for the finite element solution of frictionless contact problems, Math. Comput. Model. 28 (1998) 373-384

[29] P. Papadopoulos, R.L. Taylor, A mixed formulation for the finite element solution of contact problems, Comput. Methods Appl. Mech. Engrg. 94 (1992) 373-389.

[30] G. Pietrzak, A. Curnier, Large deformation frictional contact mechanics: continuum formulation and augmented Lagrangean treatment, Comput. Methods Appl. Mech. Engrg. 177 (1999) 351-381.

[31] A. Popp, M.W. Gee, W.A. Wall, A finite deformation mortar contact formulation using a primal-dual active set strategy, Int. J. Numer. Methods Engrg. 79 (2009) 1354-1391. 
[32] M.A. Puso, A 3D mortar method for solid mechanics, Int. J. Numer. Methods Engrg. 59 (2004) 315-336.

[33] M.A. Puso, T.A. Laursen, A mortar segment-to-segment contact method for large deformations, Comput. Methods Appl. Mech. Engrg. 193 (2004) 601-629.

[34] M.A. Puso, T.A. Laursen, A mortar segment-to-segment frictional contact method for large deformations, Comput. Methods Appl. Mech. Engrg. 193 (2004) 4891-4913.

[35] M.A. Puso, T.A. Laursen, J. Solberg, A segment-to-segment mortar contact method for quadratic elements and large deformations, Comput. Methods Appl. Mech. Engrg. 197 (2008) 555-566.

[36] J.C. Simo, Numerical analysis and simulation of plasticity, in: P.G. Ciarlet, J.L Lions (Eds.), Handbook of Numerical Analysis, vol. VI, Elsevier, 1998.

[37] J.C. Simo, P. Wriggers, R.L. Taylor, A perturbed lagrangian formulation for the finite element solution of contact problems, Comput. Methods Appl. Mech. Engrg. 50 (1985) 163-180.

[38] J.M. Solberg, R.E. Jones, P. Papadopoulos, A family of simple two-pass dual formulations for the finite element solution of contact problems, Comput Methods Appl. Mech. Engrg. 196 (2007) 782-802.

[39] J.M. Solberg, P. Papadopoulos, An analysis of dual formulations for the finite element solution of two-body contact problems, Comput. Methods Appl. Mech. Engrg. 194 (2005) 2734-2780.

[40] I. Temizer, P. Wriggers, T.J.R. Hughes, Contact treatment in isogeometric analysis with NURBS, Comput. Methods Appl. Mech. Engrg. 200 (2011) 11001112.
[41] M. Tur, F.J. Fuenmayor, P. Wriggers, A mortar-based frictional contact formulation for large deformations using Lagrange multipliers, Comput. Methods Appl. Mech. Engrg. 198 (2009) 2860-2873.

[42] K. Washizu, Variational Methods in Elasticity and Plasticity, Pergamon Press, 1968.

[43] M.F. Wheeler, T. Widley, I. Yotov, A multiscale preconditioner for stochastic mortar mixed finite elements, Comput. Methods Appl. Mech. Engrg. 200 (2011) 1251-1262.

[44] B. Wohlmuth, Variationally consistent discretization schemes and numerical algorithms for contact problems, Acta Numer. 20 (2011) 569-734.

[45] B.I. Wohlmuth, A mortar finite element method using dual spaces for the Lagrange multiplier, SIAM J. Numer. Anal. 38 (2000) 989-1012.

[46] B.I. Wohlmuth, Discretization Methods and Iterative Solvers Based on Domain Decomposition, Springer, Berlin Heidelberg New York, 2001.

[47] P. Wriggers, Computational Contact Mechanics, second ed., Springer, Berlin Heidelberg New York, 2006.

[48] B. Yang, T.A. Laursen, X. Meng, Two dimensional mortar contact methods for large deformation frictional sliding, Int. J. Numer. Methods Engrg. 62 (2005) $1183-1225$.

[49] G. Zavarise, L. De Lorenzis, R.L. Taylor, A non-consistent start-up procedure for contact problems with large load-steps, Comput. Methods Appl. Mech. Engrg. 205-208 (2012) 91-109.

[50] G. Zavarise, P. Wriggers, A superlinear convergent augmented Lagrangian procedure for contact problems, Eng. Comput. 16 (1999) 88-119. 